\title{
LQ Optimal Sliding Mode Control of Periodic Review Perishable Inventories with Transportation Losses
}

\author{
Piotr Leśniewski and Andrzej Bartoszewicz \\ Institute of Automatic Control, Technical University of Lodz, 18/22 Bohdana Stefanowskiego Street, 90-924 Lodz, Poland \\ Correspondence should be addressed to Andrzej Bartoszewicz; andrzej.bartoszewicz@p.lodz.pl
}

Received 30 July 2013; Accepted 16 September 2013

Academic Editor: Xudong Zhao

Copyright (C) 2013 P. Leśniewski and A. Bartoszewicz. This is an open access article distributed under the Creative Commons Attribution License, which permits unrestricted use, distribution, and reproduction in any medium, provided the original work is properly cited.

\begin{abstract}
In this work we apply the control-theoretic approach to design a new replenishment strategy for inventory systems with perishable stock. Such systems are supposed to effectively satisfy an unknown and permanently time-varying consumers' demand. The main obstacle of achieving this goal is the need of obtaining supplies from a distant source. During the supply process goods are inevitably lost due to various causes. Furthermore, those goods which successfully arrive at the distribution center still deteriorate while stored in its warehouse. We explicitly take into account both of these factors in designing our control strategy. We propose a sliding mode strategy and choose its parameters to minimize a quadratic quality criterion. This approach allows us to ameliorate the bullwhip effect (the amplification of the demand variations when going up in the supply chain). The control strategy proposed in this work ensures bounded orders, guarantees full consumers' demand satisfaction, and eliminates the risk of exceeding the warehouse capacity. These properties are stated in three theorems and proved in the paper.
\end{abstract}

\section{Introduction}

In a competitive economy, the problem of efficient management of supply chains has become increasingly important. Therefore, recently many attempts to solve the problem have been proposed. These attempts differ in various aspects including supply chain modeling, specific performance measures, primary objectives, and methods applied to accomplish the objectives [1]. A good overview of the results obtained in this area can be found in [2-7] and numerous references cited in those papers. The earliest application of the control theory techniques to the management of logistic processes was reported about sixty years ago when Simon (H. A. Simon for his contribution to the field received the Nobel prize in economics in 1978) in paper [8] applied servomechanism control algorithm to get a feasible strategy of goods replenishment for continuous-time, single product inventory. Soon after that a discrete time servomechanism control algorithm for managing of goods replenishment process has also been proposed [9]. Then, block diagram representation of conventional inventories and production management systems was introduced by Towill [10]. One of the most significant developments in this area was the result of Forrester [11, 12], who analyzed the amplification of demand fluctuations when moving upstream in the supply chain. The phenomenon has later been termed bullwhip effect. Important contribution to the study of this effect has been presented in [13-19]. The authors of those papers were able to smooth ordering policies and stock levels and have demonstrated that application of control theory methods helps effectively prevent the occurrence of undesirable bullwhip effect. Over the last twenty years, numerous valuable solutions in this field have been presented. Therefore, in this section we are only able to mention a few of them. In $[16,20]$, an autoregressive moving average (ARMA) system structure has been applied in order to model uncertain demand. Furthermore, model predictive control of supply chains has been widely used (see, e.g., papers [7, 21-24]), and in [25] a robust controller for the continuous-time system with uncertain processing time and delay has been designed by minimizing $\mathrm{H}$-infinity norm. Also estimation techniques have been used for the purpose of inventory management. The recursive least squares method and Kalman filter were applied in [21, 26] for delay identification and in $[16,20]$ for demand prediction, respectively. In a number of papers [27-30] the classical and modern tools of time-delay systems theory were applied to the problem of 
supply chain management. Several other methods, including convex programming [31], genetic algorithms [32], heuristic techniques [33], and simulations [34, 35], have also been applied to improve warehouse management.

In [36], state space representation of supply chains is proposed and lead time-delay is explicitly taken into account by the introduction of additional state variables. This approach results in the optimal controller designed by minimization of quadratic performance index. A similar approach is applied in [37] where an LQ optimal sliding mode controller is designed. However, both papers [36, 37] are concerned with conventional, nondeteriorating inventories only. An extension of the results presented in [36] to the case of perishable inventories is given in [38], and an LQ optimal sliding mode controller for supply chains with deteriorating stock is proposed in [39]. However, none of the papers [3639] takes into account transportation losses (or in other words goods decay during the order procurement time). Therefore, in this paper we consider perishable inventories, and we explicitly account for the ordered goods losses during the nonnegligible lead time.

In this paper, we consider a periodic-review inventory system with perishable goods replenished from a remote supply source. However, in contrast to the previously published results we consider not only losses which take place when the commodity is stored in the warehouse, but also those which happen during the supply process, that is, the losses on the way from the supplier to the warehouse. We propose a discrete time representation of the supply chain dynamics, and we apply discrete time sliding mode methodology [40$50]$ to design the controller for the considered system. The controller design objective is on the one hand to fully satisfy the imposed demand and on the other hand to ensure smoothly varying orders and in this way to avoid the bullwhip effect. This approach results in a good tradeoff between the closed loop system fast reaction to the unpredictable variations of demand and minimization of storage costs. Moreover, the sliding mode controller proposed in this paper further leads to chattering-free system operation. The controller is determined analytically in a closed form, which allows us to state and formally prove important properties of the proposed inventory policy. First, we prove that the designed management policy always generates strictly positive and upper bounded order quantities, which is an important issue from the practical point of view. Next, we define the warehouse capacity which provides enough space for all incoming shipments. Finally, we state and prove conditions ensuring that all the imposed demand is fully satisfied and $100 \%$ service level is achieved. In many practical applications, this is a desirable property directly related to customer satisfaction, and it becomes indispensable when it comes to strategic supplies (e.g., blood products).

\section{Materials and Methods}

2.1. Inventory System Model. We consider the periodic review inventory system depicted in Figure 1. At regular time instants $k T$, where $k=0,1,2, \ldots$, the stock level is checked, and a resupply order is generated. The distribution center is expected to satisfy the consumers' demand, which is modeled as an a priori unknown bounded function of time $d(k T)$, $0 \leq d(k T) \leq d_{\max }$. This is motivated by the fact that in real supply systems it is quite difficult to predict the fluctuations in consumers' demand. Therefore, our definition is quite realistic as it does not require any knowledge of the demand distribution. If the on-hand stock is insufficient, only a part of the demand is satisfied, and therefore, we introduce an additional function $h(k T)$ representing the amount of goods actually sold. Thus,

$$
0 \leq h(k T) \leq d(k T) \leq d_{\max }
$$

The distribution center is replenished from a distant source, with lead time $L$, which is a multiple of the review period, $L=$ $m T$. The quantity of goods ordered at time $k T$ is denoted by $u(k T)$. Due to transportation losses, only $\alpha u$ goods reach the warehouse (where $0 \leq \alpha \leq 1$ ) after the lead time $L$. A fraction $\sigma(0 \leq \sigma \leq 1)$ of the stock deteriorates during each review period, and to simplify the notation we introduce $p=1-\sigma$ as the part of the stock that remains unspoiled. This allows us to express the current stock level in the following form:

$$
y[(k+1) T]=p y(k T)+\alpha u[(k-m) T]-h(k T) .
$$

The amount of goods that can be sold at time $k T$ is given by

$$
h(k T)=\min (d(k T), p y(k T)+\alpha u[(k-m) T])
$$

that is, the consumer demand is covered to the extent allowed by the on-hand stock and the arriving shipments. We assume that the warehouse is initially empty $y(0)=0$, and the first order is placed at $k T=0$. Therefore, we can rewrite (2) as follows:

$$
y(k T)=\alpha \sum_{j=0}^{k-m-1} p^{k-m-1-j} u(j T)-\sum_{j=0}^{k-1} p^{k-1-j} h(j T) .
$$

In order to keep the notation as concise as possible, in the remainder of this work we will use $k$ instead of $k T$ as the independent variable.

In order to apply the control-theoretic approach to the problem considered in this paper, it is convenient to represent the supply chain model in the state space. We choose the first state variable to represent the stock level, $x_{1}(k)=y(k)$, and the remaining state variables are the delayed values of the control signal; that is,

$$
x_{j}(k)=u(k-n+j-1) \quad \text { for } j=2, \ldots, n,
$$

where $n=m+1$ is the system order. Now we can represent the system in the state space as

$$
\begin{gathered}
\mathbf{x}(k+1)=\mathbf{A} \mathbf{x}(k)+\mathbf{b} u(k)+\mathbf{o} h(k), \\
y(k)=\mathbf{q}^{T} x(k),
\end{gathered}
$$




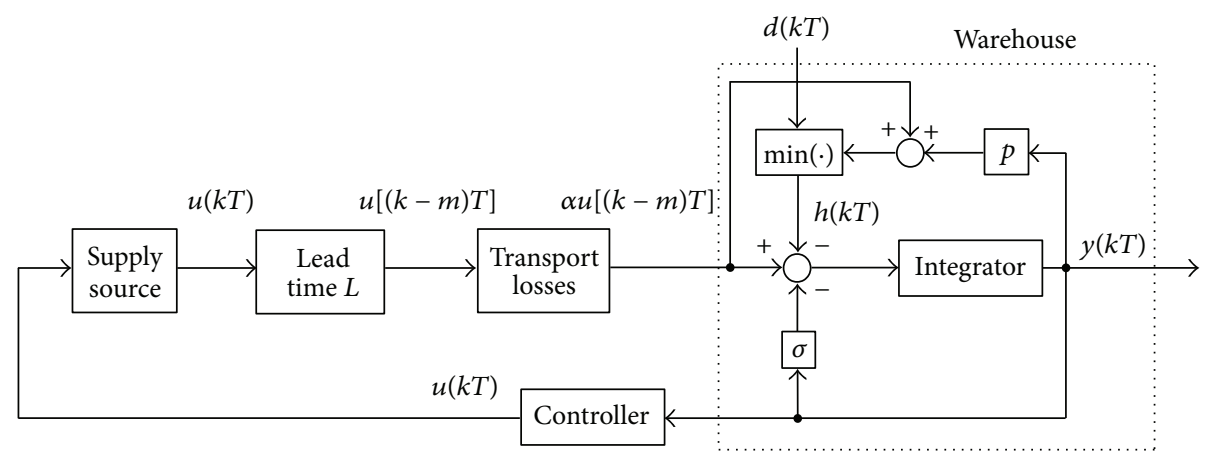

FIGURE 1: Supply chain model.

where $\mathbf{A}$ is a $n \times n$ state matrix and $\mathbf{b}, \mathbf{o}$, and $\mathbf{q}$ are $n \times 1$ vectors:

$$
\begin{gathered}
\mathbf{A}=\left[\begin{array}{ccccc}
p & \alpha & 0 & & 0 \\
0 & 0 & 1 & \cdots & 0 \\
& \vdots & & \ddots & \vdots \\
0 & 0 & 0 & \cdots & 1 \\
0 & 0 & 0 & & 0
\end{array}\right], \\
\mathbf{b}=\left[\begin{array}{c}
0 \\
0 \\
\vdots \\
0 \\
1
\end{array}\right], \quad \mathbf{o}=\left[\begin{array}{c}
-1 \\
0 \\
\vdots \\
0 \\
0
\end{array}\right], \quad \mathbf{q}=\left[\begin{array}{c}
1 \\
0 \\
\vdots \\
0 \\
0
\end{array}\right] .
\end{gathered}
$$

Due to the stock decay and transportation losses, the desired system state is given by

$$
\mathbf{x}_{d}=y_{d}\left[\begin{array}{llll}
1 & \frac{\sigma}{\alpha} & \cdots & \frac{\sigma}{\alpha}
\end{array}\right]^{T}
$$

where $y_{d}$ is the desired stock level. This completes the description of the supply chain model chosen in our work. In the following two sections we will develop a control law for the considered inventory system. We will begin with deriving a general form of the sliding mode controller. Next, we will select the parameters of the sliding hyperplane in order to minimize an LQ criterion. Then, we will formulate and prove important properties of the proposed control strategy.

2.2. Sliding Mode Controller Design. We introduce a sliding hyperplane $s(k)=0$ described by the following equation:

$$
s(k)=\mathbf{c}^{T} \mathbf{e}(k)
$$

where $\mathbf{c}^{T}=\left[c_{1} c_{2} \ldots c_{n}\right]$ is a vector satisfying $\mathbf{c}^{T} \mathbf{b} \neq 0$ and $\mathbf{e}(k)=$ $\mathbf{x}_{d}-\mathbf{x}(k)$ is the closed loop system error. By substituting (6) into $\mathbf{c}^{T} \mathbf{e}(k+1)=0$ the following feedback control law can be derived:

$$
u(k)=\left(\mathbf{c}^{T} \mathbf{b}\right)^{-1} \mathbf{c}^{T}\left[\mathbf{x}_{d}-\mathbf{A x}(k)\right] .
$$

Using (7) we can present (10) as follows:

$$
\begin{array}{r}
u(k)=c_{n}^{-1}\left[y_{d}\left(c_{1}+\frac{\sigma}{\alpha} \sum_{i=2}^{n} c_{i}\right)-c_{1} p y(k)\right. \\
\left.-c_{1} \alpha x_{2}(k)-\sum_{i=3}^{n} c_{i-1} x_{i}(k)\right] .
\end{array}
$$

With the application of this control strategy, the state matrix of the closed loop system has the form $\mathbf{A}_{c}=\left[\mathbf{I}_{n}-\mathbf{b}\left(\mathbf{c}^{T} \mathbf{b}\right)^{-1} \mathbf{c}^{T}\right] \mathbf{A}$. The characteristic polynomial of $\mathbf{A}_{c}$ can be found as follows:

$$
\begin{aligned}
\operatorname{det}\left(z \mathbf{I}_{n}-\mathbf{A}_{c}\right)= & z^{n}+z^{n-1} \frac{c_{n-1}-p c_{n}}{c_{n}} \\
& +z^{n-2} \frac{c_{n-2}-p c_{n-1}}{c_{n}}+\cdots+z \frac{c_{1} \alpha-p c_{2}}{c_{n}},
\end{aligned}
$$

and the design may be completed by the pole placement method. However, as the pole placement does not directly lead to optimal performance, in this work we select the parameters $c_{1}, c_{2}, \ldots, c_{n}$ by minimizing a quadratic quality criterion.

2.3. Optimization Problem. We seek for a control signal $u_{\text {opt }}(k)$ that will steer the system towards the desired state, while providing smooth responses to the consumers' demand changes. This goal can be formulated as the minimization of the following cost functional:

$$
J(u)=\sum_{k=0}^{\infty}\left[u^{2}(k)+\mathbf{e}^{T}(k) \mathbf{W e}(k)\right] .
$$

We select $\mathbf{W}=\operatorname{diag}\left(w_{1}, w_{2}, \ldots, w_{2}\right)$, where $w_{1}$ and $w_{2}$ are positive weighting factors adjusting the impact of on the one hand the stock level error and on the other hand the deviation of the recent orders from the steady state value $y_{d} \sigma / \alpha$, respectively. As stated in [51] the control that minimizes (13) is given by

$$
u_{\mathrm{opt}}(k)=r-\mathbf{g x}(k)
$$


where

$$
\mathbf{g}=\mathbf{b}^{*} \mathbf{K}\left(\mathbf{I}_{\mathbf{n}}+\mathbf{b} \mathbf{b}^{*} \mathbf{K}\right)^{-1} \mathbf{A}
$$

and $r$ is a constant term. Operator $(\cdot)^{*}$ denotes the complex conjugate matrix transpose, and matrix $\mathbf{K}$ is semipositive definite and satisfies $\mathbf{K}^{*}=\mathbf{K}$. It can be found by solving the following Riccati equation:

$$
\mathbf{K}=\mathbf{A}^{*} \mathbf{K}\left(\mathbf{I}_{\mathbf{n}}+\mathbf{b} \mathbf{b}^{*} \mathbf{K}\right)^{-1} \mathbf{A}+\mathbf{W} .
$$

Since all elements of $\mathbf{A}$ and $\mathbf{b}$ are real numbers, the complex conjugate matrix transpose is equivalent to the matrix transpose $(\cdot)^{T}$. Consequently, all elements of $\mathbf{K}$ are also real numbers, and condition $\mathbf{K}^{*}=\mathbf{K}$ implies that $\mathbf{K}$ is symmetric. Therefore, we will represent all elements of $\mathbf{K}$ positioned below the main diagonal by " $*$ " in order to simplify the notation.

As stated in the previous chapter the system order $n$ depends on the supply lead time. Because of this and in order to draw general conclusions we must solve (16) analytically for an arbitrary system order. The method applied here consists of iterative substitution of $\mathbf{K}$ into the expression on the right hand side of (16) and comparison with its left hand side. This allows us to reduce the number of independent elements of $\mathbf{K}$ in each iteration and finally to solve (16).

The first step involves substituting the most general form of $\mathbf{K}$ into (16). This yields

$$
\mathbf{K}_{\mathbf{1}}=\left[\begin{array}{ccccc}
k_{11} & \frac{\alpha}{p}\left(k_{11}-w_{1}\right) & k_{13} & & k_{1 n} \\
* & \frac{\alpha^{2}}{p^{2}}\left(k_{11}-w_{1}\right)+w_{2} & k_{23} & \cdots & k_{2 n} \\
* & * & k_{33} & & k_{3 n} \\
& \vdots & & \ddots & \vdots \\
* & * & * & \cdots & k_{n n}
\end{array}\right] .
$$

In the next iteration, we substitute (17) into (16) and compare the resulting matrices. We repeat this procedure, until every element of $\mathbf{K}$ is expressed as a function of $k_{11}$, the weighting factors $w_{1}$ and $w_{2}$, the system order $n$, and the system parameters $\alpha$ and $p$. At this stage we get

$$
\begin{aligned}
& \mathbf{K}= \\
& {\left[\begin{array}{cccc}
k_{11} & \frac{\alpha}{p}\left(k_{11}-w_{1}\right) & & \frac{\alpha}{p^{n-1}}\left(k_{11}-w_{1} \sum_{i=0}^{n-2} p^{2 i}\right) \\
* & \frac{\alpha^{2}}{p^{2}}\left(k_{11}-w_{1}\right)+w_{2} & \cdots & \frac{\alpha^{2}}{p^{n}}\left(k_{11}-w_{1} \sum_{i=0}^{n-2} p^{2 i}\right) \\
& \vdots & \ddots & \vdots \\
* & * & \cdots & \frac{\alpha^{2}}{p^{2 n-2}}\left(k_{11}-w_{1} \sum_{i=0}^{n-2} p^{2 i}\right)+(n-1) w_{2}
\end{array}\right] .}
\end{aligned}
$$

In order to obtain the final form of matrix $\mathbf{K}$, we determine $k_{11}$ by substituting (18) into (16) and examining the first elements on the left and right hand sides. This yields the following equation:

$$
\begin{gathered}
\alpha^{2} k_{11}^{2}-k_{11}\left\{w_{1} \alpha^{2}\left(2-p^{2 n-2}+2 p^{2} \frac{1-p^{2 n-2}}{1-p^{2}}\right)\right. \\
\left.-p^{2 n-2}\left(1-p^{2}\right)\left[1+(n-1) w_{2}\right]\right\} \\
+\frac{w_{1}^{2} \alpha^{2}\left(1-p^{2 n}-p^{2 n-2}+p^{4 n-2}\right)}{\left(1-p^{2}\right)^{2}} \\
+p^{2 n-2} w_{1}\left[1+(n-1) w_{2}\right]=0 .
\end{gathered}
$$

The above equation has two roots

$$
\begin{aligned}
k_{11}^{ \pm}= & \left\{w_{1} \alpha^{2} \frac{2-p^{2 n}-p^{2 n-2}}{1-p^{2}}-p^{2 n-2}\left(1-p^{2}\right)\right. \\
& \left.\times\left[1+(n-1) w_{2}\right] \pm p^{2 n-2} \sqrt{\Delta}\right\}\left(2 \alpha^{2}\right)^{-1},
\end{aligned}
$$

where

$$
\begin{aligned}
\Delta= & w_{1}^{2} \alpha^{4}+2 \alpha^{2} w_{1}\left[1+(n-1) w_{2}\right]\left(p^{2}+1\right) \\
& +\left(1-p^{2}\right)^{2}\left[1+(n-1) w_{2}\right]^{2},
\end{aligned}
$$

but only $k_{11}^{+}$guarantees that $\mathbf{K}$ is semipositive definite. Having found $\mathbf{K}$, we calculate vector $\mathbf{g}$. For that purpose, we substitute $k_{11}^{+}$into (15), and we get

$$
\mathbf{g}=\gamma\left[\begin{array}{lllll}
\alpha^{-1} & p^{-1} & p^{-2} & \cdots & p^{-n+1}
\end{array}\right],
$$

where

$$
\gamma=p^{n}\left\{1-\frac{2\left[1+(n-1) w_{2}\right]}{w_{1} \alpha^{2}+\left[1+(n-1) w_{2}\right]\left(1+p^{2}\right)+\sqrt{\Delta}}\right\} .
$$

Taking into account (5), we conclude that the control signal that minimizes (13) can be expressed as

$$
u_{\mathrm{opt}}(k)=r-\frac{\gamma y(k)}{\alpha}-\gamma p^{-n} \sum_{i=k-m}^{k-1} p^{k-i} u(i) .
$$

The derived control law corresponds to the following choice of vector $\mathbf{c}$ :

$$
\mathbf{c}=\left[\begin{array}{lllll}
\gamma p^{-1} \alpha^{-1} & \gamma p^{-2} & \cdots & \gamma p^{-n+1} & 1
\end{array}\right]^{T} .
$$

Using (25) with (11) and comparing the result with (24), we find that the constant term $r$ is given by

$$
r=y_{d} \alpha^{-1}\left(1-p+\gamma p^{-n+1}\right) .
$$

This completes the design of the LQ optimal sliding mode controller. Next, we will present and prove important properties of the proposed controller. 
2.4. Stability Analysis. The design method described before ensures the closed loop system stability. In this subsection we verify this property and find the closed loop poles. The characteristic polynomial of the system with the application of the proposed control law is given by

$$
\operatorname{det}\left(z \mathbf{I}_{\mathbf{n}}-\mathbf{A}_{\mathbf{c}}\right)=z^{n-1}\left[z-p\left(1-\gamma p^{-n}\right)\right]
$$

where $\mathbf{A}_{\mathbf{c}}=\left[\mathbf{I}_{\mathbf{n}}-\mathbf{b}\left(\mathbf{c}^{T} \mathbf{b}\right)^{-1} \mathbf{c}^{T}\right] \mathbf{A}$ is the closed loop state matrix. We notice that all roots but one of (27) lie in the origin of the $z$-plane. We also observe from (23) that $0<\gamma<p^{n}$. Therefore, the remaining one root is located on the real axis between 0 and $p$. Since $p \leq 1$, we conclude that the system is in fact stable, and no oscillations appear in the system.

Remark 1. By selecting different parameters, $w_{1}$ and $w_{2}$, the proposed control law can be tuned to meet specific users' requirements. When $w_{1} \rightarrow \infty$, then for any finite $w_{2}$ the output error dominates the quality criterion, the controller becomes a dead-beat scheme, and its gain $\gamma$ approaches $p^{n}$. On the other hand, when $w_{1} \rightarrow 0$ the influence of the output error diminishes and gain $\gamma$ drops to zero. Moreover, as the states $x_{2}, x_{3}, \ldots, x_{n}$ are the delayed values of the control signal, decreasing $w_{2}$ leads to an increase of $\gamma$ and vice versa.

2.5. Properties of the Proposed Control Strategy. We will now formulate and prove important properties of the proposed control law. In the first theorem, we show that the order quantities generated by the controller are always upper and lower bounded. The second one demonstrates that the onhand stock level never exceeds its demand value, and the last theorem determines the minimum demand stock size that ensures $100 \%$ consumers' demand satisfaction.

Theorem 2. The order quantities generated by the proposed control law are always bounded by the following inequalities:

$$
\frac{y_{d} \sigma}{\alpha} \leq u(k) \leq \max \left(r, \frac{y_{d} \sigma}{\alpha}+\frac{\gamma d_{\max }}{\alpha\left[1+p\left(\gamma p^{-n}-1\right)\right]}\right) .
$$

Proof. We notice from (24) that $u(0)=r$. This means that (28) is satisfied for $k=0$. By substituting (4) into (24) we can obtain

$$
u(k)=r-\frac{\gamma}{\alpha}\left[\alpha \sum_{j=0}^{k-1} p^{k-m-1-j} u(j)-\sum_{j=0}^{k-1} p^{k-1-j} h(j)\right] .
$$

We will now assume that (28) holds for all integers up to some $l \geq 0$ and demonstrate that this implies that (28) is also true for $l+1$. We can express (29) for $k=l+1$ as follows:

$$
\begin{aligned}
u(l+1)= & r-\gamma p^{-m} u(l)+\frac{\gamma}{\alpha} h(l) \\
& -\frac{\gamma}{\alpha}\left[\alpha \sum_{j=0}^{l-1} p^{l-m-j} u(j)-\sum_{j=0}^{l} p^{l-j} h(j)\right] \\
= & r-\gamma p^{-m} u(l)+\frac{\gamma}{\alpha} h(l)-p[r-u(l)] \\
= & r(1-p)+p\left(1-\gamma p^{-n}\right) u(l)+\frac{\gamma h(l)}{\alpha} .
\end{aligned}
$$

Using (26) and taking into account that $h(k) \geq 0$, we can derive the minimum value of $u(l+1)$

$$
u(l+1) \geq \frac{y_{d} \sigma}{\alpha}
$$

which means that the first inequality in (28) always holds. Because $h(k) \leq d_{\max }$ for any $k \geq 0$, we can find the maximum value of $u(k)$ :

$$
u(l+1) \leq r(1-p)+p\left(1-\gamma p^{-n}\right) u(l)+\frac{\gamma d_{\max }}{\alpha} .
$$

We will begin our analysis with the case

$$
r \geq \frac{y_{d} \sigma}{\alpha}+\frac{\gamma d_{\max }}{\alpha\left[1+p\left(\gamma p^{-n}-1\right)\right]} .
$$

Taking into consideration (26) and (33) we arrive at

$$
\frac{\gamma d_{\max }}{\alpha} \leq r \gamma p^{-n+1} .
$$

Using this inequality with (32) we obtain

$$
\begin{aligned}
u(l+1) & \leq r(1-p)+p\left(1-\gamma p^{-n}\right) r+\frac{\gamma d_{\max }}{\alpha} \\
& =r-\gamma p^{-n+1} r+\frac{\gamma d_{\max }}{\alpha} \leq r .
\end{aligned}
$$

Now, we will consider the second case, that is, the situation when

$$
r<\frac{y_{d} \sigma}{\alpha}+\frac{\gamma d_{\max }}{\alpha\left[1+p\left(\gamma p^{-n}-1\right)\right]} .
$$

Because $u(l)$ satisfies (28), substituting (26) and (36) into (32) we get

$$
\begin{aligned}
u(l+1) \leq & \frac{y_{d}}{\alpha}\left(1-p+\gamma p^{-n+1}\right)(1-p)+\left(p-\gamma p^{-n+1}\right) \\
& \times\left\{\frac{y_{d} \sigma}{\alpha}+\frac{\gamma d_{\max }}{\alpha\left[1+p\left(\gamma p^{-n}-1\right)\right]}\right\}+\frac{\gamma d_{\max }}{\alpha} \\
= & \frac{y_{d} \sigma}{\alpha}+\frac{\gamma d_{\max }}{\alpha\left(1+\gamma p^{-n+1}-p\right)} .
\end{aligned}
$$

Using the principle of mathematical induction with relations (31), (35), and (37) we conclude that (28) is indeed true for any $k \geq 0$. This ends the proof. 
In supply systems, it is important to ensure that all incoming shipments can be accommodated in the distribution center, as the cost of external emergency storage is usually extremely high. In the next theorem, we show that for our strategy a warehouse size equal to the demand stock size is sufficient to guarantee this property.

Theorem 3. With the application of the proposed control strategy the on-hand stock never exceeds its demand value, that is, for any $k \geq 0$

$$
y(k) \leq y_{d}
$$

Proof. As the warehouse is empty for any $k \leq n-1$, we only need to demonstrate that (38) holds for $k \geq n$. We will start by assuming that (38) holds for some integer $l \geq n$ and show that this implies that it is also true for $l+1$. Using (29) with (2) we obtain

$$
\begin{aligned}
y(l+1)= & p y(l)+\alpha r-\gamma p^{-n} \sum_{j=l-m}^{l-1} p^{l-j} h(j)-h(l) \\
& -\gamma p^{-m}\left[\alpha \sum_{j=0}^{l-m-1} p^{l-m-1-j} u(j)-\sum_{j=0}^{l-1} p^{l-1-j} h(j)\right] .
\end{aligned}
$$

From (4), we can observe that the last term in (39) is equal to $\gamma p^{-m} y(l)$. Therefore,

$$
\begin{aligned}
y(l+1)= & \alpha r+p y(l)\left(1-\gamma p^{-n}\right) \\
& -\gamma p^{-n} \sum_{j=l-m}^{l-1} p^{l-j} h(j)-h(l) .
\end{aligned}
$$

Using relations $h(k) \geq 0,(26)$, and the assumption $y(l) \leq y_{d}$, we arrive at

$$
y(l+1) \leq y_{d}\left(1-p+\gamma p^{-n+1}\right)+y_{d}\left(p-\gamma p^{-n+1}\right)=y_{d} .
$$

As already stated in the previous section, $y(k)=0$ for $k<n$. Furthermore, since (23) implies $\gamma \leq p^{n}$, we can observe from (26) that $r \leq y_{d} / \alpha$. Therefore, the stock size for $k=n$, which can be obtained from (2), always satisfies

$$
y(n)=\alpha r-h(m) \leq y_{d}
$$

Using the principle of mathematical induction with (41) and (42), we conclude that (38) is true for $k \geq n$. As already stated, $y(k)=0$ for $k<n$; therefore, (38) holds for any $k \geq 0$.

According to Theorem 3, if a storage capacity of $y_{d}$ is secured at the warehouse, then all incoming shipments will be accommodated. This means that the risk of high cost connected with emergency storage is eliminated.

Theorem 4. If the proposed control strategy is applied and the reference stock level satisfies the following inequality:

$$
y_{d}>\frac{\gamma\left(1-p^{-m}\right)+p-1}{(p-1)\left(1-p+\gamma p^{-n+1}\right)} d_{\max },
$$

then for any $k \geq n$, the on-hand stock level is strictly positive.

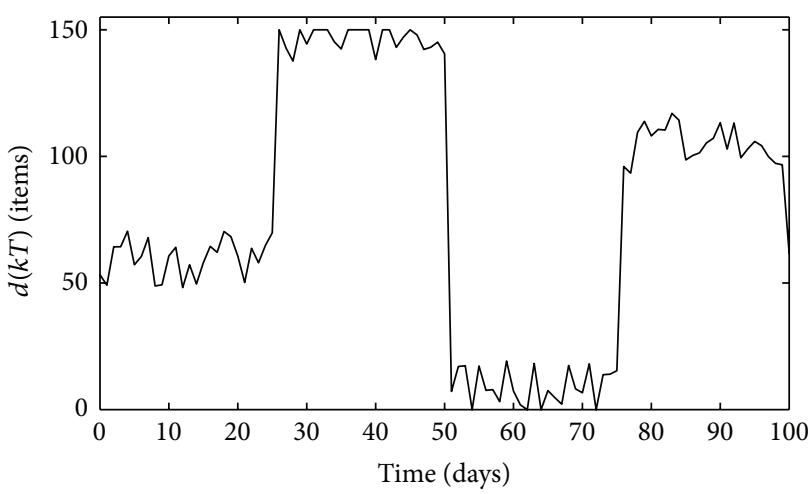

Figure 2: Consumers demand.

Proof. We will demonstrate that if (43) is satisfied, then for any $l \geq m$ condition $y(l)>0$ implies $y(l+1)>0$. Because $h(k) \leq d_{\max }$ for any $k>0$, we can obtain for $l \geq m$ the following inequality from (40):

$$
\begin{aligned}
y(l+1) \geq & \alpha y_{d} \alpha^{-1}\left(1-p+\gamma p^{-n+1}\right)+p y(l)\left(1-\gamma p^{-n}\right) \\
& -d_{\max }\left(1+\gamma p^{-n} \sum_{j=l-m}^{l-1} p^{l-j}\right) \\
\geq & y_{d}\left(1-p+\gamma p^{-n+1}\right)-d_{\max }\left(1+\gamma \frac{1-p^{-m}}{p-1}\right)>0 .
\end{aligned}
$$

We can observe from (42) that if (43) is satisfied, then the stock size for $k=m+1=n$ is strictly positive. Using this observation and (44) using the principle of mathematical induction, we conclude that if (43) is satisfied, then the stock level is indeed positive for any $k \geq m+1$.

As we can observe from (2), if the stock level in period $k$ is strictly positive, then the demand in period $k-1$ has been fully satisfied. The last theorem shows that our control strategy ensures the full satisfaction of demand $d(k)$ for any $k \geq m$.

\section{Results and Discussion}

In order to present the properties of the proposed control strategy, computer simulations are performed. We select the review period $T$ as 1 day, and the lead time $L$ is assumed as 7 days. This implies that $m=7$ and $n=8$. The consumer demand, which is depicted in Figure 2, exhibits rapid changes between small and large values. This reflects the most difficult conditions that could arise in the system. It is assumed that transportation losses are equal to $4 \%$, which implies that $\alpha=0.96$. The on-hand stock deteriorates at rate $\sigma=0.02$, which corresponds to $p=0.98$.

We select the weighting factor $w_{2}$ as 0.4 and perform three simulations, each with a different value of $w_{1}$. For each case, we compute gain $\gamma$ from (23) and the minimum demand stock value $y_{d}^{\prime}$ that ensures full demand satisfaction from (43). For each simulation scenario we use a slightly larger demand 
TABLE 1: Simulation parameters.

\begin{tabular}{lccc}
\hline$w_{1}$ & $\gamma$ & $y_{d}^{\prime}$ (items) & $y_{d}$ (items) \\
\hline 10 & 0.6433 & 1306 & 1315 \\
1 & 0.3178 & 1468 & 1475 \\
0.1 & 0.1086 & 2015 & 2025 \\
\hline
\end{tabular}

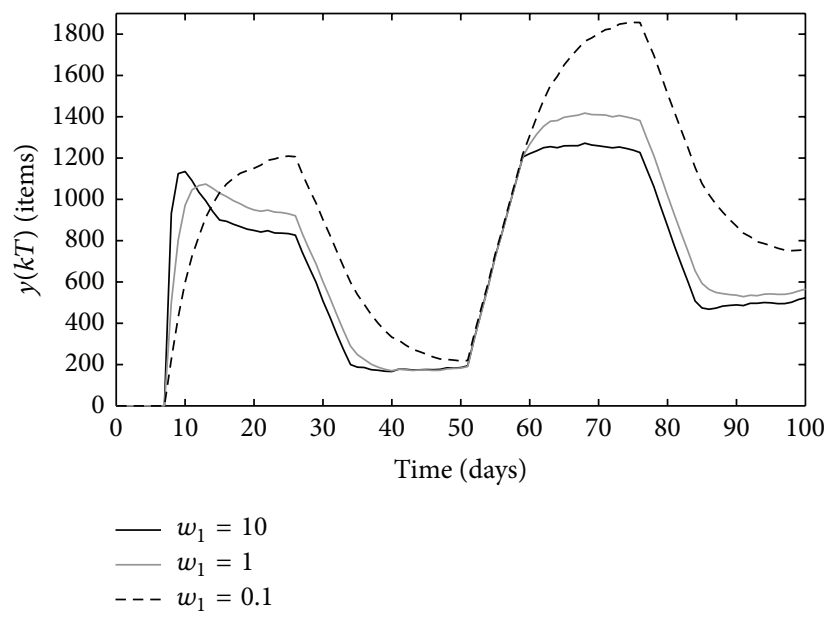

FIgURE 3: On-hand stock level for different values of $w_{1}$.

stock size denoted by $y_{d}$. The parameters mentioned above are given in Table 1.

Figures 3 and 4 show the results of the simulations. The value of the control signal at the beginning of the control process is shown in Figure 5. As we can observe, the proposed controller always generates upper and lower bounded replenishment orders, as predicted by Theorem 2 . Moreover, the on-hand stock never exceeds its demand value and never decreases to zero for $k \geq m+1$. Therefore, all incoming shipments can always be accommodated in the warehouse, and the consumer demand is fully covered.

We can tune the algorithm to meet specific needs by choosing appropriate values of $w_{1}$ and $w_{2}$, which changes the value of $\gamma$. High $\gamma$ results in faster tracking of consumers' demand, which means that smaller warehouse capacity is sufficient to ensure full demand satisfaction. On the other hand smaller values of $\gamma$ smoothen out the control signal and therefore diminish the bullwhip effect.

\section{Conclusions}

In this paper, we proposed an optimal sliding mode controller for periodic review inventory systems. During the design we have taken into account not only the decay of goods while stored in the warehouse, but also the losses which may occur during transport. We have demonstrated that the proposed control strategy always generates upper and lower bounded resupply orders and ensures that all incoming shipments can be stored in the allocated warehouse space. Furthermore, we derived the minimum size of the warehouse that ensures full consumer demand satisfaction.

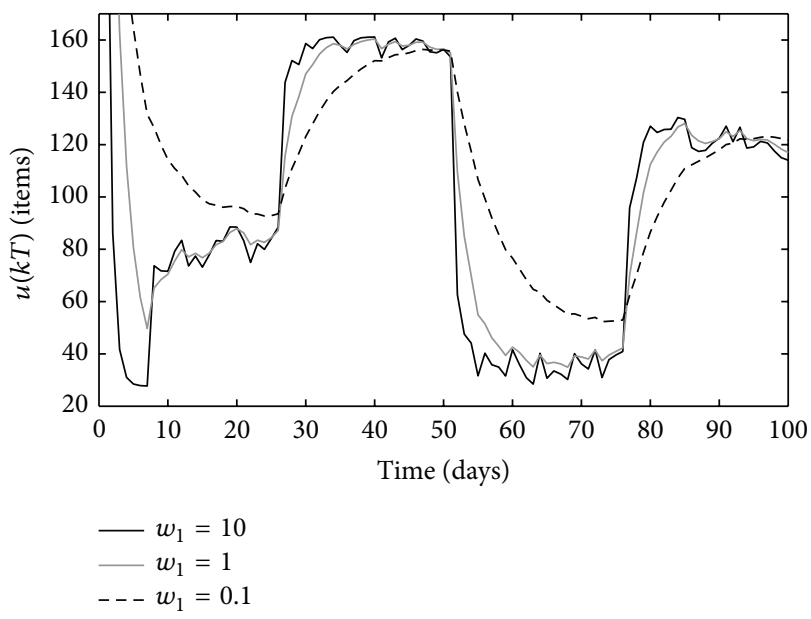

FIGURE 4: Replenishment orders for different values of $w_{1}$.

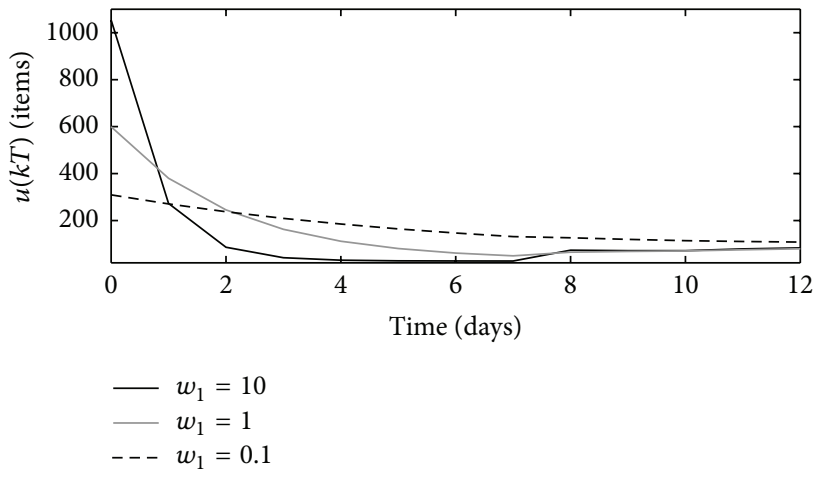

FIGURE 5: Replenishment orders at the beginning of the control process.

\section{Conflict of Interests}

The authors declare that there is no conflict of interests regarding the publication of this paper.

\section{Acknowledgments}

This work has been performed in the framework of a project "Optimal Sliding Mode Control of Time Delay Systems" financed by the National Science Centre of Poland, Decision no. DEC 2011/01/B/ST7/02582. Kind support provided by the Foundation for Polish Science under Mistrz grant is also acknowledged.

\section{References}

[1] B. M. Beamon, "Supply chain design and analysis: models and methods," International Journal of Production Economics, vol. 55, no. 3, pp. 281-294, 1998.

[2] H. Sarimveis, P. Patrinos, C. D. Tarantilis, and C. T. Kiranoudis, "Dynamic modeling and control of supply chain systems: a review," Computers and Operations Research, vol. 35, no. 11, pp. 3530-3561, 2008. 
[3] C. E. Riddalls, S. Bennett, and N. S. Tipi, "Modelling the dynamics of supply chains," International Journal of Systems Science, vol. 31, no. 8, pp. 969-976, 2000.

[4] I. Karaesmen, A. Scheller-Wolf, and B. Deniz, "Managing perishable and aging inventories: review and future research directions," in Handbook of Production Planning, K. Kempf, P. Keskinocak, and R. Uzsoy, Eds., Kluwer, Dordrecht, The Netherlands, 2008.

[5] M. Boccadoro, F. Martinelli, and P. Valigi, "Supply chain management by $\mathrm{H}$-infinity control," IEEE Transactions on Automation Science and Engineering, vol. 5, no. 4, pp. 703-707, 2008.

[6] K. Hoberg, J. R. Bradley, and U. W. Thonemann, "Analyzing the effect of the inventory policy on order and inventory variability with linear control theory," European Journal of Operational Research, vol. 176, no. 3, pp. 1620-1642, 2007.

[7] K. Subramanian, Integration of control theory and scheduling methods for supply chain management [Ph.D. thesis], University of Wisconsin, Madison, Wis, USA, 2013.

[8] H. A. Simon, "On the application of servomechanism theory in the study of production control," Econometrica, vol. 20, no. 2, pp. 247-268, 1952.

[9] H. J. Vassian, Application of Discrete Variable Servo Theory to Inventory Control, Arthur D. Little, Cambridge, Mass, USA, 1954.

[10] D. R. Towill, "Dynamic analysis of an inventory and order based production control system," International Journal of Production Research, vol. 20, no. 6, pp. 671-687, 1982.

[11] J. Forrester, "Industrial dynamics, a major breakthrough for decision makers," Harvard Business Review, vol. 36, no. 4, pp. 37-66, 1958.

[12] J. Forrester, Industrial Dynamics, MIT Press, Cambridge, Mass, USA, 1961.

[13] J. Dejonckheere, S. M. Disney, M. R. Lambrecht, and D. R. Towill, "The impact of information enrichment on the Bullwhip effect in supply chains: a control engineering perspective," European Journal of Operational Research, vol. 153, no. 3, pp. 727-750, 2003.

[14] S. M. Disney and D. R. Towill, "On the bullwhip and inventory variance produced by an ordering policy," Omega, vol. 31, no. 3, pp. 157-167, 2003.

[15] S. M. Disney and D. R. Towill, "A methodology for benchmarking replenishment-induced bullwhip," Supply Chain Management, vol. 11, no. 2, pp. 160-168, 2006.

[16] G. Gaalman and S. M. Disney, "State space investigation of the bullwhip problem with ARMA $(1,1)$ demand processes," International Journal of Production Economics, vol. 104, no. 2, pp. 327-339, 2006.

[17] C. S. Lalwani, S. M. Disney, and D. R. Towill, "Controllable, observable and stable state space representations of a generalized order-up-to policy," International Journal of Production Economics, vol. 101, no. 1, pp. 172-184, 2006.

[18] A. Potter, D. Towill, T. Böhme, and S. Disney, “The influence of multi-product production strategy on factory induced bullwhip," International Journal of Production Research, vol. 47, no. 20, pp. 5739-5759, 2009.

[19] L. Zhou, S. Disney, and D. R. Towill, "A pragmatic approach to the design of bullwhip controllers," International Journal of Production Economics, vol. 128, no. 2, pp. 556-568, 2010.

[20] G. Gaalman, "Bullwhip reduction for ARMA demand: the proportional order-up-to policy versus the full-state-feedback policy," Automatica, vol. 42, no. 8, pp. 1283-1290, 2006.
[21] E. Aggelogiannaki, P. Doganis, and H. Sarimveis, "An adaptive model predictive control configuration for productioninventory systems," International Journal of Production Economics, vol. 114, no. 1, pp. 165-178, 2008.

[22] X. Li and T. E. Marlin, "Robust supply chain performance via Model Predictive Control," Computers and Chemical Engineering, vol. 33, no. 12, pp. 2134-2143, 2009.

[23] W. Wang, D. E. Rivera, and K. G. Kempf, "Model predictive control strategies for supply chain management in semiconductor manufacturing," International Journal of Production Economics, vol. 107, no. 1, pp. 56-77, 2007.

[24] M. W. Braun, D. E. Rivera, M. E. Flores, W. M. Carlyle, and K. G. Kempf, "A Model Predictive Control framework for robust management of multi-product, multi-echelon demand networks," Annual Reviews in Control, vol. 27, pp. 229-245, 2003.

[25] E. K. Boukas, P. Shi, and R. K. Agarwal, "An application of robust control technique to manufacturing systems with uncertain processing time," Optimal Control Applications and Methods, vol. 21, no. 6, pp. 257-268, 2000.

[26] E. Aggelogiannaki and H. Sarimveis, "Design of a novel adaptive inventory control system based on the online identification of lead time," International Journal of Production Economics, vol. 114, no. 2, pp. 781-792, 2008.

[27] C. E. Riddalls and S. Bennett, "The stability of supply chains," International Journal of Production Research, vol. 40, no. 2, pp. 459-475, 2002.

[28] R. Sipahi and I. I. Delice, "Stability of inventory dynamics in supply chains with three delays," International Journal of Production Economics, vol. 123, no. 1, pp. 107-117, 2010.

[29] R. D. H. Warburton, S. M. Disney, D. R. Towill, and J. P. E. Hodgson, "Further insights into the stability of supply chains," International Journal of Production Research, vol. 42, no. 3, pp. 639-648, 2004.

[30] I. Delice, Stability analysis of multiple time-delay systems with applications to supply chain management [Ph.D. thesis], Northeastern University, 2011.

[31] Y. Feng, S. Chen, A. Kumar, and B. Lin, "Solving single-product economic lot-sizing problem with non-increasing setup cost, constant capacity and convex inventory cost in $\mathrm{O}(\mathrm{N} \log \mathrm{N})$ time," Computers and Operations Research, vol. 38, no. 4, pp. 717-722, 2011.

[32] S. H. R. Pasandideh, S. T. A. Niaki, and A. R. Nia, "A genetic algorithm for vendor managed inventory control system of multi-product multi-constraint economic order quantity model," Expert Systems with Applications, vol. 38, no. 3, pp. 2708-2716, 2011.

[33] S.-C. Liu and J.-R. Chen, "A heuristic method for the inventory routing and pricing problem in a supply chain," Expert Systems with Applications, vol. 38, no. 3, pp. 1447-1456, 2011.

[34] P. Köchel and U. Nieländer, "Simulation-based optimisation of multi-echelon inventory systems," International Journal of Production Economics, vol. 93-94, pp. 505-513, 2005.

[35] A. Tal and T. Arponen, "An EOQ model for items with a fixed shelf-life and a declining demand rate based on time-to-expiry technical note," Asia-Pacific Journal of Operational Research, vol. 26, no. 6, pp. 759-767, 2009.

[36] P. Ignaciuk and A. Bartoszewicz, "Linear-quadratic optimal control strategy for periodic-review inventory systems," Automatica, vol. 46, no. 12, pp. 1982-1993, 2010. 
[37] P. Ignaciuk and A. Bartoszewicz, "LQ optimal sliding mode supply policy for periodic review inventory systems," IEEE Transactions on Automatic Control, vol. 55, no. 1, pp. 269-274, 2010.

[38] P. Ignaciuk and A. Bartoszewicz, "Linear-quadratic optimal control of periodic-review perishable inventory systems," IEEE Transactions on Control Systems Technology, vol. 20, no. 5, pp. 1400-1407, 2012.

[39] P. Ignaciuk and A. Bartoszewicz, "LQ optimal sliding-mode supply policy for periodic-review perishable inventory systems," Journal of the Franklin Institute, vol. 349, no. 4, pp. 15611582, 2012.

[40] V. Utkin and S. V. Drakunow, "On discrete-time sliding mode control," IFAC Conference on Nonlinear Control, pp. 484-489, 1989.

[41] K. Furuta, "Sliding mode control of a discrete system," Systems and Control Letters, vol. 14, no. 2, pp. 145-152, 1990.

[42] W. Gao, Y. Wang, and A. Homaifa, "Discrete-time variable structure control systems," IEEE Transactions on Industrial Electronics, vol. 42, no. 2, pp. 117-122, 1995.

[43] A. Bartoszewicz, "Remarks on 'Discrete-time variable structure control systems", IEEE Transactions on Industrial Electronics, vol. 43, no. 1, pp. 235-238, 1996.

[44] G. Golo and C. Milosavljević, "Robust discrete-time chattering free sliding mode control," Systems and Control Letters, vol. 41, no. 1, pp. 19-28, 2000.

[45] B. Bandyopadhyay and S. Janardhanan, "Discrete-time sliding mode control: a multirate output feedback approach," Lecture Notes in Control and Information Sciences, vol. 323, pp. 1-151, 2005.

[46] C. Milosavljević, B. Peruničić-Draženović, B. Veselić, and D. Mitić, "Sampled data quasi-sliding mode control strategies," IEEE International Conference on Industrial Technology, pp. 2640-2645, 2006.

[47] S. Janardhanan and B. Bandyopadhyay, "Multirate output feedback based robust quasi-sliding mode control of discrete-time systems," IEEE Transactions on Automatic Control, vol. 52, no. 3, pp. 499-503, 2007.

[48] S. J. Mija and S. Thomas, "Reaching law based sliding mode control for discrete MIMO systems," in Proceedings of the 11th International Conference on Control, Automation, Robotics and Vision (ICARCV '10), pp. 1291-1296, December 2010.

[49] M. L. Corradini, V. Fossi, A. Giantomassi, G. Ippoliti, S. Longhi, and G. Orlando, "Discrete time sliding mode control of robotic manipulators: Development and experimental validation," Control Engineering Practice, vol. 20, no. 20, pp. 816-822, 2012.

[50] X. Yu, B. Wang, and X. Li, "Computer-controlled variable structure systems: the state-of-the-art," IEEE Transactions on Industrial Informatics, vol. 8, no. 2, pp. 197-205, 2012.

[51] H. Kwakernaak and R. Sivan, Linear Optimal Control Systems, Wiley-Interscience, New York, NY, USA, 1972. 


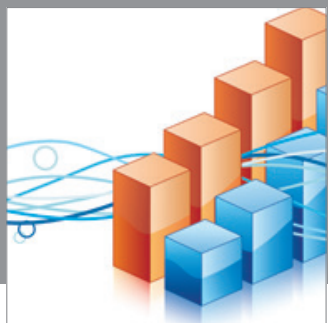

Advances in

Operations Research

mansans

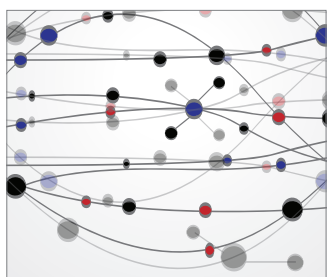

The Scientific World Journal
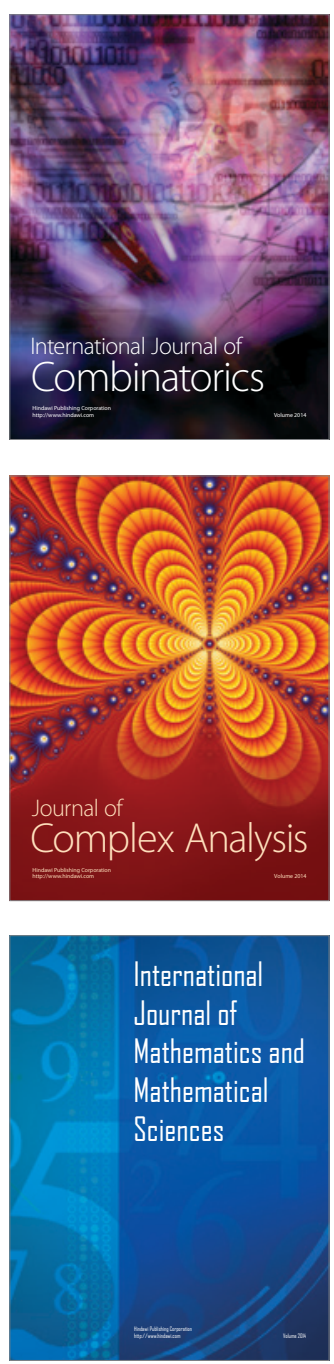
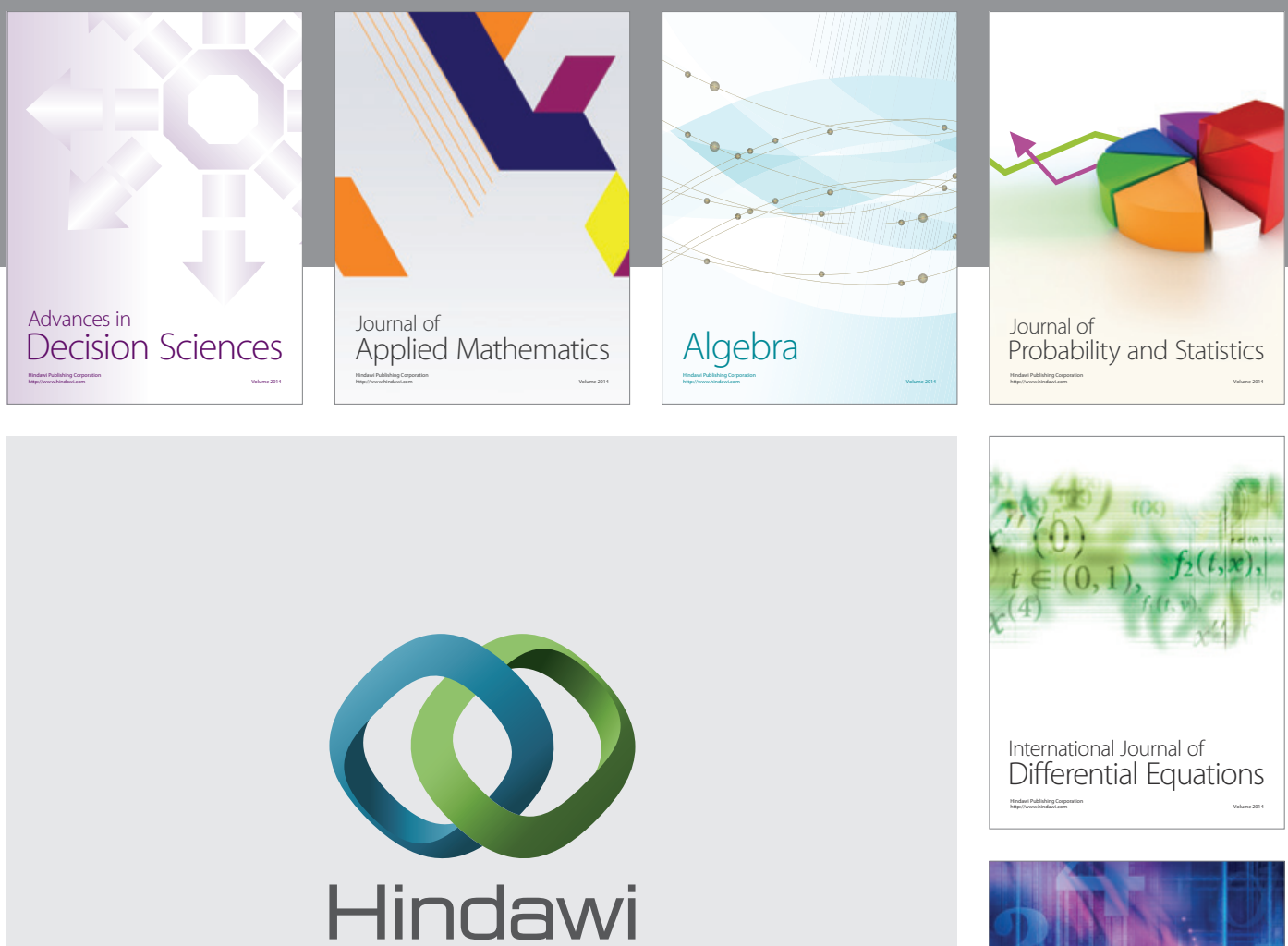

Submit your manuscripts at http://www.hindawi.com
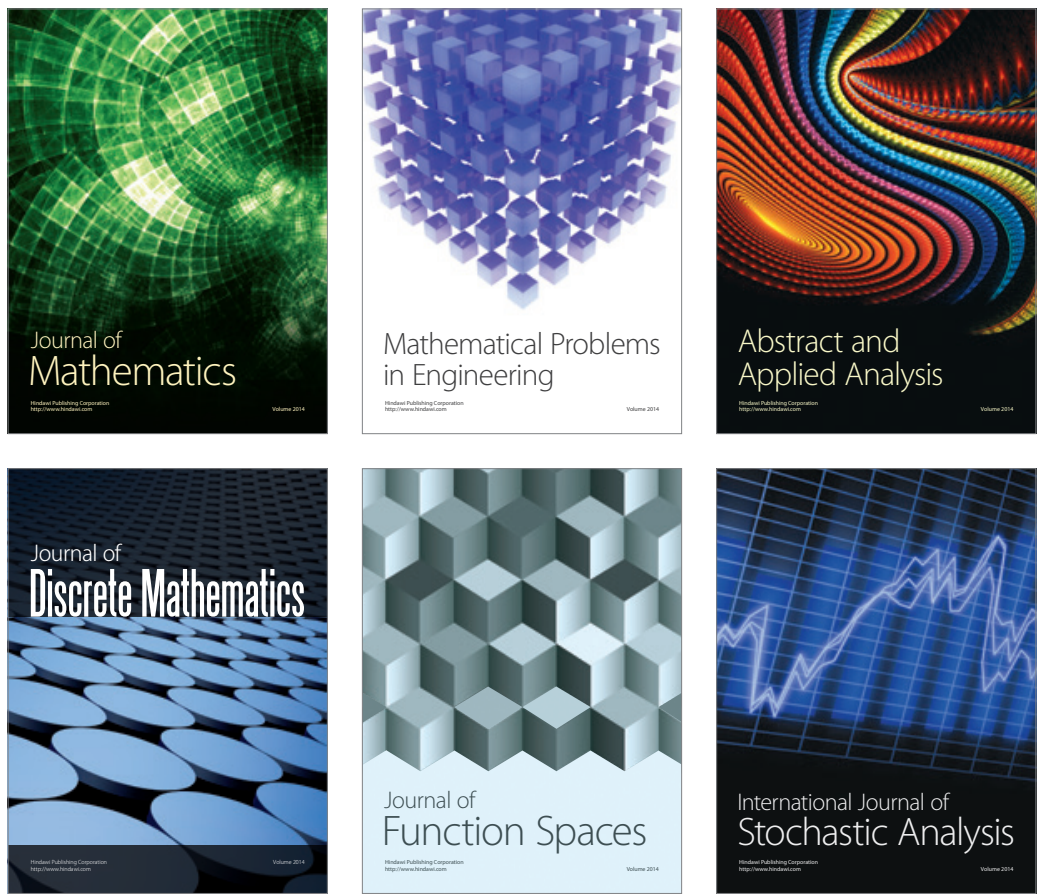

Journal of

Function Spaces

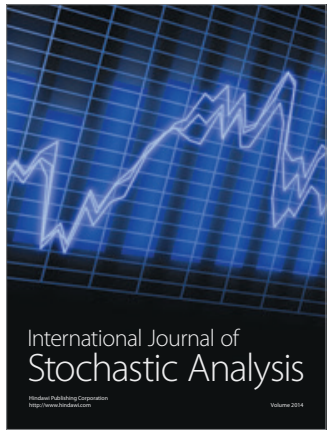

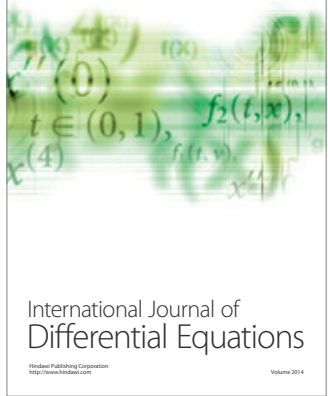
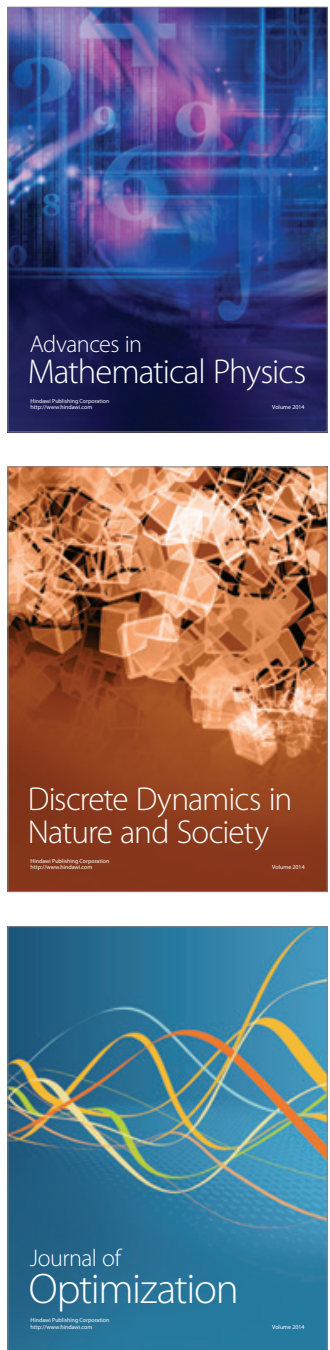